\title{
Topological effects associated with fractional statistics in one-dimensional mesoscopic rings
}

\author{
Jian-Xin Zhu and Z. D. Wang \\ Department of Physics, University of Hong Kong, Pokfulam Road, Hong Kong
}

(Received 7 February 1995)

\begin{abstract}
Using both the continuum and tight-binding models, we have investigated analytically the effects of a statistical phase factor on the persistent charge and magnetic moment currents of "free" hard-core anyons in one-dimensional mesoscopic rings. In particular, we find that the ground state of the system could, in most cases, self-sustain the Aharonov-Bohm and Aharonov-Casher effects simultaneously.
\end{abstract}

PACS number(s): 03.65.Bz, 66.90+r

The importance of quantum effects in mesoscopic systems, such as the persistent current occurring in isolated mesoscopic normal metal rings penetrated by an AharonovBohm [1-4] (AB) flux, has been widely recognized. Considering that such a persistent current itself also produces magnetic flux in addition to the flux from external sources, the possibility of a self-sustained persistent current in the absence of external flux was recently addressed [5]. A ground state with such a spontaneous current is known as a flux-phase state [6]. It is also interesting to note that there exists another topological interference effect-the AharonovCasher (AC) effect [7], which is the electromagnetic dual of the $\mathrm{AB}$ effect; a particle with a magnetic moment $\boldsymbol{\mu}$ moving in an electric field $\mathscr{E}$ acquires an AC phase [8] due to the spin-orbit interaction. Thus the spin- $\frac{1}{2}$ electrons in a mesoscopic metal ring pierced by a charged rod are expected to display a persistent spin current [9], which is periodic in the AC flux $\Phi_{A C} \equiv(\mu / e) \oint_{C} \mathscr{E} \times \hat{\mathbf{z}} \cdot d \boldsymbol{l}$. This AC effect has also been discussed for a fluxon in type-II superconductors [10] and for a vortex in Josephson-junction arrays [11]. In addition, based on a similar idea for the spontaneous AB effect, an interesting spontaneous AC effect for electrons in a onedimensional (1D) mesoscopic normal-metal ring was investigated more recently $[12,13]$. In identifying the existence of spontaneous topological effects, the statistics of the particles plays a crucial role. On the other hand, the concept of fractional statistics or anyons [14], has been a subject of intense study over the past several years. Such entities may be of great importance in the study of the fractional quantum Hall effect [15] and high-temperature superconductivity $[14,16]$. However, it has been realized that calculations incorporating general anyons present rather unique and challenging problems, even if their only interaction is due to their statistics [17]. Fortunately, the formalism of hard-core anyons can be well constructed, since in this case the anyon world lines do not cross and the notion of braids still holds [18]. Notice that the anyon concept is essentially two dimensional; a recent study [19] of spinon excitations in one-dimensional antiferromagnets, however, has suggested that anyons could exist in one-dimensional systems. Thus a fundamental and important question naturally arises: What will happen to the topological effects for 1D anyons? In this paper, within the framework of the continuum and tight-binding models, we study the persistent currents due to the $\mathrm{AB}$ and $\mathrm{AC}$ effects in a $1 \mathrm{D}$ meso- scopic ring of "free" hard-core anyons having both charge and magnetic moment. In particular, the interesting spontaneous $\mathrm{AB}$ and $\mathrm{AC}$ effects are proposed.

Let us first consider $N_{a}$ free hard-core anyons confined to a 1D ring with radius $R$ (circumference $L=2 \pi R$ ), vanishingly small width $b$, and thickness $l$, lying in $x y$ plane. In the presence of both $\mathrm{AB}$ flux and $\mathrm{AC}$ flux [20], the total flux $\Phi=\Phi_{\mathrm{AB}}+\Phi_{\mathrm{AC}}$. The continuum free hard-core anyon model is defined by the following four conditions.

(i) The wave function satisfies the Schrödinger equation for the motion of $N_{a}$ free anyons, each possessing charge $q$ and magnetic moment $\mu$, in the ring.

(ii) The wave function satisfies the phase condition with respect to the interchange of particle coordinates

$$
\Psi\left(x_{i j}^{\prime}, \ldots\right)=-e^{-i \chi \pi} \Psi\left(x_{i j}, \ldots\right)
$$

here $x_{i j}^{\prime}=e^{i \pi} x_{i j}$ is any two-particle relative position $x_{i j}=x_{i}-x_{j}$ rotated through a positive angle $\pi$ not enclosing any other particles. The parameter $\chi$ indicates that we are dealing with fractional statistics, and for fermions we have $\chi=0$ while for bosons $\chi=1$ [21].

(iii) The wave function satisfies the cyclic boundary condition, which in the presence of both the $\mathrm{AB}$ and $\mathrm{AC}$ fluxes reads in part

$$
\Psi\left(x_{1}+L, x_{2}, \ldots, x_{N_{a}}\right)=e^{i 2 \pi f} \Psi\left(x_{1}, x_{2}, \ldots, x_{N_{a}}\right),
$$

with a similar condition for the derivative. Here $f=f_{\mathrm{AB}}+f_{\mathrm{AC}}=\Phi_{\mathrm{AB}} / \Phi_{0}+\Phi_{\mathrm{AC}} / \Phi_{0}$ with the flux quantum $\Phi_{0}=h c / e$. The direction of the magnetic moment carried by each anyon is assumed to be parallel to the positive $z$ axis.

(iv) The wave function vanishes when the coordinates of two particles coincide.

In consideration of conditions (i), (ii), and (iv), the wave function in any region should have the form [22]

$$
\Psi=\left(e^{-i \chi \pi}\right)^{P} \operatorname{det}\left|e^{i k_{n} x_{j}}\right|,
$$

where $\left(e^{-i \chi \pi}\right)^{P}$ denotes the statistical phase resulting from the permutation and $P$ is the number of transpositions of two variables that brings any other region to the region 
$R_{1}:\left(0 \leqslant x_{1} \leqslant x_{2} \leqslant \cdots \leqslant x_{N_{a}} \leqslant L\right)$. Using the boundary condition Eq. (2), we can find the energy $E_{n}$ of the $n$th eigenstate

$$
E_{n}=\frac{\hbar^{2}}{2 m_{a} R^{2}}(f+n+\bar{\chi})^{2}
$$

where $m_{a}$ is the anyon mass, $n=0, \pm 1, \pm 2, \ldots$, and $\bar{\chi}=\left(N_{a}-1\right) \chi / 2-\operatorname{Int}\left[\left(N_{a}-1\right) \chi / 2\right]$. Note that, from Eq. (3), each eigenstate can be occupied by only one anyon. The energy of $N_{a}$ anyons is given by the sum of Eq. (4) over all occupied levels

$$
E=\frac{\hbar^{2}}{2 m_{a} R^{2}}\left[\sum_{n} n^{2}+2(f+\bar{\chi}) \sum_{n} n+N_{a}(f+\bar{\chi})^{2}\right]
$$

Defining

$$
\nu= \begin{cases}\bar{\chi} & \text { for odd } N_{a} \\ \bar{\chi}-1 / 2 & \text { for even } N_{a}\end{cases}
$$

we can write the ground-state energy of $N_{a}$ anyons in a compact form, i.e.,

$$
E=E_{0}^{(o, e)}+\frac{\hbar^{2} N_{a}}{2 m_{a} R^{2}}(f+\nu)^{2}
$$

where $E_{0}^{(o, e)}$ are the $f$-independent energy constants corresponding to odd and even number of particles and $f$ is in the range $[-1 / 2-\nu, 1 / 2-\nu)$. Once the ground-state energy is known, the persistent charge (magnetic moment) current is then given by the derivative of the energy $E$ with respect to $f_{\mathrm{AB}}\left(f_{\mathrm{AC}}\right)[23,24]$, i.e.,

$$
I_{p}=\frac{N_{a} \hbar p}{2 m_{a} \pi R^{2}}(f+\nu)
$$

where $p=q, \mu$ represent the charge and magnetic moment currents, respectively. Clearly, both of the persistent currents in each period vary linearly with the total flux and there is a discontinuous jump at the end of each period.

So far we have not taken into account the flux $\Phi_{I}$ induced by the charge and magnetic moment currents themselves. From physical considerations, this self-induced flux should be included. In particular, at sufficiently low temperatures and small external driven flux $\Phi_{\text {ext }}, \Phi_{I}$ can become of the order of $\Phi_{\text {ext }}$ or even larger. This raises the possibility of a self-sustained flux in 1D anyon systems. According to Faraday's law $\boldsymbol{\nabla} \times \mathscr{E}=-(1 / c)(\partial \mathbf{B} / \partial t)$, we can easily obtain the AC flux generated by the magnetic moment current and the corresponding energy stored in this electric field $[12,13]$

$$
f_{\mathrm{AC}}^{(I)}=-\frac{4 \pi R \mu}{l b \hbar c^{2}} I_{\mu}, \quad E_{\mathscr{C}}=\frac{l b \hbar^{2} c^{2}}{4 R \mu^{2}} f_{\mathrm{AC}}^{2}
$$

Furthermore, the charge current $I_{q}$ induces an AB flux and produces the corresponding magnetic energy $[5,12]$

$$
f_{\mathrm{AB}}^{(I)}=-\frac{\mathscr{b} c}{(e / q) \Phi_{0}} I_{q}, \quad E_{B}=\frac{(e / q)^{2} \Phi_{0}^{2}}{2 \mathscr{b} c^{2}} f_{\mathrm{AB}}^{2},
$$

where $\mathscr{B}=4 \pi R^{2} / l c^{2}$ is the self-inductance of the ring. In the absence of the external AB and AC fluxes, Eqs. (8)-(10) lead to a set of self-consistent solutions

$$
f_{\mathrm{AB}}^{(s)}=-\frac{\nu B}{A+B+A B}, \quad f_{\mathrm{AC}}^{(s)}=-\frac{\nu A}{A+B+A B},
$$

where $A \equiv 4 \pi^{2} m_{a} R^{2} / N_{a} q^{2} \mathscr{B}$ and $B \equiv m_{a} c^{2} R l b / 2 N_{a} \mu^{2}$.

On the other hand, the total energy of the system is the sum of the energy of $N_{a}$ anyons and the energy stored in the magnetic and electric fields, i.e., $E_{T}=E+E_{B}+E_{\mathscr{E}}$. It is straightforward to find that $E_{T}$ reaches its minimum just at $f_{\mathrm{AB}}=f_{\mathrm{AB}}^{(s)}$ and $f_{\mathrm{AC}}=f_{\mathrm{AC}}^{(s)}$, which implies that as long as $\nu \neq 0$ the state with spontaneous $\mathrm{AB}$ and $\mathrm{AC}$ fluxes is a stable ground state of the system and therefore the time-reversal symmetry is spontaneously broken. Indeed, we do have $\nu \neq 0$ for a general hard-core anyon gas $(0<\chi<1)$. Also note that when $\chi=0, \nu=0$ for an odd $N_{a}$ but $\nu=-1 / 2$ for an even $N_{a}$, which implies that the spontaneous $\mathrm{AB}$ and $\mathrm{AC}$ fluxes could only be trapped by a ring with an even number of particles; when $\chi=1, \nu=0$ for both odd and even $N_{a}$, which results in no self-sustained flux state regardless of the number of particles $N_{a}$ being even or odd [25]. Consequently, Eq. (11) recovers the results for the spontaneous effects in the two special cases, i.e., 1D normal-metal rings $[12,13]$ and $1 \mathrm{D}$ rings of a neutral hard-core boson gas [26]. For semions $(\chi=1 / 2)$, one finds that $\nu=1 / 4,-1 / 4,1 / 2$ for $N_{a}=4 m_{0}, 4 m_{0}+2,4 m_{0}+3$ while $\nu=0$ for $N_{a}=4 m_{0}+1$, which indicates that there exist spontaneous effects in 1D semion rings, except when $N_{a}=4 m_{0}+1$. Since an anyon possesses both charge and magnetic moment, the charge current accompanies magnetic moment current and therefore the spontaneous $\mathrm{AB}$ and $\mathrm{AC}$ effects cannot be separated in $1 \mathrm{D}$ anyon rings. The relative strength of the two fluxes is determined by the factor $A / B=2 \mu^{2} / q^{2} R b$. If $A \ll B$ (e.g., electronic systems) then $f_{\mathrm{AC}}^{(s)} \approx 0$ and $f_{\mathrm{AB}}^{(s)}=\nu$ so that the selfconsistent flux comes almost purely from the anyonic statistics. Nevertheless, for general anyons, $A / B$ may not be definitely small because the magnetic moment $\mu$ and charge $q$ depend on the properties of anyons considered. If we assume that an anyon carries charge $q=e$ and flux $\phi=\Phi_{0} / 2$ and take typically mesoscopic values $R \sim 10^{-4} \mathrm{~cm}$ and $l \sim b \sim 10^{-7} \mathrm{~cm}$, we can find $A / B \sim 2$. Here $\mu=\phi l / 4 \pi$ has been used.

At this stage, it is also of importance and interest to study the topological effects by using the tight-binding model. The second quantized Hamiltonian of the model reads

$$
H=-\sum_{i=1}^{N}\left(t_{i+1, i} a_{i+1}^{\dagger} a_{i} e^{i \Delta \theta_{i}}+\text { H.c. }\right)+\sum_{i=1}^{N} \epsilon_{i} a_{i}^{\dagger} a_{i},
$$

where $t_{i j}$ is the hopping matrix element, $\epsilon_{i}$ characterizes the on-site energy, and $\Delta \theta_{i}$ are the phases acquired by anyons hopping successively from the $i$ th site to the $(i+1)$ th site and they obey

$$
\sum_{i}^{N} \Delta \theta_{i}=2 \pi f
$$


$a_{i}^{\dagger}$ and $a_{i}$ construct a set of anyon creation and annihilation operators defined on the sites and satisfy the generalized equal-time commutation relations [18]

$$
a_{i} a_{j}^{\dagger}=\delta_{i j}-e^{-i \chi \pi} a_{j}^{\dagger} a_{i}, \quad a_{i} a_{j}=\delta_{i j}-e^{i \chi \pi} a_{j} a_{i}
$$

for $i \neq j$ and the hard-core condition

$$
a_{i} a_{i}=a_{i}^{\dagger} a_{i}^{\dagger}=0
$$

To solve the eigenvalue problem of $H$ in Eq. (12), it is crucial to perform the generalized Jordan-Wigner transformation

$$
\begin{gathered}
a_{j}=\exp \left(i \chi \pi \sum_{1 \leqslant s<j} c_{s}^{\dagger} c_{s}\right) c_{j}, \\
a_{j}^{\dagger}=c_{j}^{\dagger} \exp \left(-i \chi \pi \sum_{1 \leqslant s<j} c_{s}^{\dagger} c_{s}\right),
\end{gathered}
$$

where the operators $c_{j}^{\dagger}$ and $c_{j}$ obey the standard anticommutation relations. Then we can arrive at an equivalent Hamiltonian

$$
\begin{aligned}
H= & \sum_{i=1}^{N} \epsilon_{i} c_{i}^{\dagger} c_{i}-\sum_{i=1}^{N-1}\left(t_{i+1, i} c_{i+1}^{\dagger} c_{i} e^{i \Delta \theta_{i}}+\text { H.c. }\right) \\
& -\left(t_{1 N} c_{1}^{\dagger} c_{N} e^{i\left[\Delta \theta_{N}+\chi \pi\left(N_{a}-1\right)\right]}+\text { H.c. }\right),
\end{aligned}
$$

which describes the quantum motion of a system of $N_{a}$ fermions coupled to a "fictitious" gauge field in addition to the vector and scalar potentials. Note that the periodic boundary condition $a_{1}=a_{N+1}$ has been used here. In general, all $\theta$ 's are different even for the perfect lattice (i.e., $t_{i j}=t$, $\epsilon_{i}=$ const), but physical quantities should globally depend on the total flux enclosed by the ring. This fact becomes quite clear when we perform a unitary transformation $U_{i j}=\exp \left[-i\left(\sum_{l=i}^{N} \Delta \theta_{l}-\Delta \theta_{N}\right)\right] \delta_{i j} \quad[27]$, which simplifies the Hamiltonian given by Eq. (15) in the single-site representation to

$$
\tilde{H}=\left(\begin{array}{cccc}
\epsilon_{1} & -t_{12} & \cdots & -\tilde{t}_{1 N}^{*} \\
-t_{12} & \epsilon_{2} & \cdots & \\
\cdots & \ldots & \ldots & \ldots \\
-\tilde{t}_{1 N} & & \ldots & \epsilon_{N}
\end{array}\right)
$$

with $\tilde{t}_{1 N}=t_{1 N} e^{-i \pi\left[2 f+\chi\left(N_{a}-1\right)\right]}$. For the perfect lattice (set $\left.\epsilon_{i} \equiv 0\right)$, performing a further unitary transformation $\bar{U}_{j j^{\prime}}=e^{i(N-j) \Delta \tilde{\theta}} \delta_{j j^{\prime}}$ with $\Delta \tilde{\theta}=(2 \pi / N)(f+\bar{\chi})$ to redistribute phases uniformly, followed by the well-known Fourier transformation, the energy of the $n$th eigenstate can be obtained analytically

$$
E_{n}=-2 t \cos \left[\frac{2 \pi}{N}(f+n+\bar{\chi})\right]
$$

At zero temperature, the ground-state energy of $N_{a}$ anyons and the persistent current are given by

$$
E=-\varepsilon_{0} \cos \left[\frac{2 \pi}{N}(f+\nu)\right]
$$

and

$$
I_{p}=\frac{p \varepsilon_{0}}{N \hbar} \sin \left[\frac{2 \pi}{N}(f+\nu)\right]
$$

respectively, where $\varepsilon_{0}=2 t \sin \left(N_{a} \pi / N\right) / \sin (\pi / N)$. Obviously, for large $N$ (with fixed $N_{a} / N$ and lattice constant) the expressions for the currents in the tight-binding model are similar to the continuum formulas.

In a similar way, one can obtain the spontaneous $\mathrm{AB}$ and $\mathrm{AC}$ fluxes. In the limit of vanishingly small cross section of the ring, they can be approximated as

$$
f_{\mathrm{AB}}^{(s)} \approx-\frac{\nu B^{\prime}}{A^{\prime}+B^{\prime}+A^{\prime} B^{\prime}}, \quad f_{\mathrm{AC}}^{(s)} \approx-\frac{\nu A^{\prime}}{A^{\prime}+B^{\prime}+A^{\prime} B^{\prime}},
$$

where $A^{\prime} \equiv N^{2} \hbar^{2} / q^{2} \varepsilon_{0} \mathscr{C}$ and $B^{\prime} \equiv N^{2} \hbar^{2} c^{2} l b / 8 \pi^{2} \mu^{2} \varepsilon_{0} R$. Note that $A^{\prime} / B^{\prime}=2 \mu^{2} / q^{2} R b$, which is the same as that in the continuum model. Moreover, a similar discussion and conclusions, concerning spontaneous topological effects, can be made in the present case.

It is worthwhile to point out here that all hard-core anyons are exponentially localized in one-dimensional disordered systems. However, for weak disorder, the characteristic localization length could be greater than the typical size of the mesoscopic ring [2]. We thus expect that the spontaneous topological effects will survive in these systems. At finite temperatures, one can similarly define a characteristic temperature $T^{*}$ [e.g., $T^{*}=\left(2 t / k_{B} \pi N\right) \sin \left(N_{a} \pi / N\right)$ [2] for the tight-binding model]. The persistent current decreases dramatically with $T / T^{*}$ at $T>T^{*}$; however, it is insensitive to temperature at $T<T^{*}$. Finally, we wish to mention that, in general, the Coulomb repulsion between anyons needs to be included unless their charge $q$ is small enough. As we have shown, the 1D problem of $N_{a}$ hard-core anyons is equivalent to $N_{a}$ fermions coupled to a fictitious gauge field. With this analogy, several results obtained for a system of fermions apply to the present case. Numerical calculations by either the exact-diagonalization technique [28] or the Hartree-Fock approximation [29] for small-size normal metal rings have indicated that both long-range and short-range interactions suppress the amplitude of persistent currents. However, the flux dependence of the current does not change qualitatively. Therefore, it is rather reasonable to conclude that the spontaneous topological effects could still exist in the system mentioned with the Coulomb interaction included, as long as the self-induced flux is taken into account.

To summarize, we have calculated the persistent charge and magnetic moment currents for hard-core anyons confined to 1D mesoscopic rings, with particular emphasis on the effect of the statistical phase factor. In general, both of the spontaneous $\mathrm{AB}$ and $\mathrm{AC}$ effects could simultaneously occur in $1 \mathrm{D}$ rings of hard-core anyons.

We are grateful to Dr. P. K. MacKeown for a critical reading of our manuscript. This work was supported by the RGC grant of Hong Kong under Grant No. HKU 262/95P and a CRCG research grant at the University of Hong Kong. 
[1] Y. Aharonov and D. Bohm, Phys. Rev. 115, 485 (1959).

[2] Ho-Fai Cheung, Yuval Gefen, E. K. Riedel, and Wei-Heng Shih, Phys. Rev. B 37, 6050 (1988).

[3] L. P. Lévy, G. Dolan, J. Dunsmuir, and H. Bouchiat, Phys. Rev. Lett. 64, 2074 (1990); V. Chandrasekhar, R. A. Webb, M. J. Brady, M. B. Ketchen, W. J. Gallagher, and A. Kleinsasser, ibid. 67, 3578 (1991); D. Mially, C. Chapelier, and A. Benoit, ibid. 70, 2020 (1993).

[4] Jian-Xin Zhu and Z. D. Wang, Phys. Rev. B 50, 7207 (1994).

[5] D. Wolleben, M. Esser, P. Freche, E. Zipper, and M. Szopa, Phys. Rev. Lett. 66, 3191 (1991); Mod. Phys. Lett. B 6, 1481 (1992).

[6] V. Kalmeyer and R. B. Laughlin, Phys. Rev. Lett. 59, 2095 (1987); I. Affleck and J. B. Marston, Phys. Rev. B 37, 3774 (1988).

[7] Y. Aharonov and A. Casher, Phys. Rev. Lett. 53, 319 (1984); C. R. Hagen, ibid. 64, 2347 (1990).

[8] Sometimes the phase obtained by a fluxon encircling a charge (see Ref. [10] below) is also called the AC phase, however, this name seems more appropriate for the case of a point magnetic dipole moving around a line of charge.

[9] A. V. Balatsky and B. L. Altshuler, Phys. Rev. Lett. 70, 1678 (1993).

[10] R. Reznik and Y. Aharonov, Phys. Rev. D 40, 4178 (1989).

[11] B. J. van Wees, Phys. Rev. Lett. 65, 255 (1990); T. P. Orlando and K. A. Delin, Phys. Rev. B 43, 8717 (1991); W. J. Elion, J. J. Wachters, L. L. Sohn, and J. E. Mooij, Phys. Rev. Lett. 71, 2311 (1993).

[12] M. Y. Choi, Phys. Rev. Lett. 71, 2987 (1993).

[13] Jian-Xin Zhu and Z. D. Wang, J. Phys.: Condens. Matter 6, L329 (1994).

[14] Fractional Statistics and Anyon Superconductivity, edited by F. Wilczek (World Scientific, Singapore, 1989); Physics and Mathematics of Anyons, edited by S. S. Chern, C. W. Chu, and
C. S. Ting (World Scientific, Singapore, 1991).

[15] B. I. Halperin, Phys. Rev. Lett. 52, 1583 (1983); 52, 2390(E) (1984); D. Arovas, J. R. Schrieffer, and F. Wilczek, ibid. 53, 722 (1984); S. M. Girvin and A. H. MacDonald, ibid. 58, 1252 (1987).

[16] R. Laughlin, Science 242, 525 (1988); A. L. Fetter, C. B. Hanna, and R. B. Laughlin, Phys. Rev. B 39, 9679 (1989).

[17] See, e.g., S. A. Chin and C.-R. Hu, Phys. Rev. Lett. 69, 229 (1992).

[18] E. Fradkin, Field Theories of Condensed Matter Systems (Addison-Wesley, Redwood City, CA, 1991).

[19] F. D. M. Haldane, Phys. Rev. Lett. 66, 1529 (1991).

[20] As demonstrated in Ref. [10], the interaction term $\mathscr{E} \times \boldsymbol{\mu}$, although appearing as a local interaction, actually represents a nonlocal interaction.

[21] Here we work with a flux tube attached to a charged fermion to compose an anyon. If a flux tube is attached to a charged boson, as treated in Ref. [17], simply by replacing $\chi$ with $1-\alpha$, all results derived in our paper are the same.

[22] E. H. Lieb and W. Liniger, Phys. Rev. 130, 1605 (1963); M. Girardeau, J. Math. Phys. 1, 516 (1960); Jian-Xin Zhu and Z. D. Wang, Phys. Rev. B 51, 13813 (1995).

[23] N. Byers and C. N. Yang, Phys. Rev. Lett. 7, 46 (1961).

[24] F. Bloch, Phys. Rev. B 2, 109 (1970).

[25] If the antiperiodic condition is chosen, which is not unreasonable when we insert a $\pi$-phase-shift junction in the ring, there will exist spontaneous $\mathrm{AB}$ and $\mathrm{AC}$ fluxes regardless of the number of particles being even or odd.

[26] Jian-Xin Zhu, Z. D. Wang, and Qin-Wei Shi, J. Phys. A 27, L875 (1995).

[27] Z. D. Wang and Jian-Xin Zhu, Phys. Rev. B 52, 5275 (1995).

[28] M. Abraham and R. Berkovites, Phys. Rev. Lett. 70, 1509 (1993); G. Bouzerar, D. Poiblanc, and G. Montambaux, Phys. Rev. B 49, 8258 (1994).

[29] H. Kato and D. Yoshioka, Phys. Rev. B 50, 4943 (1994). 